\title{
A novel device for left atrial appendage exclusion
}

Keiji Kamohara, MD, ${ }^{a}$ Kiyotaka Fukamachi, MD, PhD, ${ }^{a}$ Yoshio Ootaki, MD, PhD, ${ }^{\text {a }}$ Masatoshi Akiyama, MD, PhD, ${ }^{\text {a }}$ Firas Zahr, MD, ${ }^{a}$ Michael W. Kopcak, Jr, BA, ${ }^{a}$ Raymond Dessoffy, AA, ${ }^{a}$ Zoran B. Popović, MD, ${ }^{\text {b }}$ Masao Daimon, MD, Delos M. Cosgrove, MD, ${ }^{\mathrm{c}}$ and $\mathrm{A}$. Marc Gillinov, $\mathrm{MD}^{\mathrm{c}}$

From the Departments of Biomedical Engineering, ${ }^{\text {a }}$ Lerner Research Institute; Cardiovascular Medicine, ${ }^{\mathrm{b}}$ and Thoracic and Cardiovascular Surgery, ${ }^{\mathrm{c}}$ The Cleveland Clinic Foundation, Cleveland, Ohio.

Supported by AtriCure, Inc (Cincinnati, Ohio).

Received for publication May 18, 2005; revisions received Aug 11, 2005; accepted for publication Aug 15, 2005.

Address for reprints: Kiyotaka Fukamachi, $\mathrm{MD}, \mathrm{PhD}$, Department of Biomedical Engineering/ND20, The Cleveland Clinic Foundation, 9500 Euclid Ave, Cleveland, $\mathrm{OH}$ 44195 (E-mail: fukamak@ccf.org).

J Thorac Cardiovasc Surg 2005;130:1639-44 $0022-5223 / \$ 30.00$

Copyright (C) 2005 by The American Association for Thoracic Surgery

doi:10.1016/j.jtcvs.2005.08.019
Objective: The left atrial appendage is a frequent source of thromboemboli in patients with atrial fibrillation. Exclusion or excision of the left atrial appendage may reduce the risk of stroke in patients with atrial fibrillation. We evaluated the ability of a novel device to exclude the left atrial appendage during early and intermediate follow-up periods in a canine model.

Methods: Eight mongrel dogs (mean weight $29.1 \pm 4.0 \mathrm{~kg}$ ) were used in this study. The occlusion device, constructed from 2 stainless steel strips covered with a knit braided polyester fabric, was implanted at the base of the left atrial appendage through a left thoracotomy on a beating heart. Dogs were evaluated at 7 days $(n=2)$, 30 days $(n=2)$, and 90 days $(n=4)$ by epicardial echocardiography, left atrial angiography, histologic inspection, and gross pathology.

Results: Device implantation was performed without complications in all animals. Complete exclusion of the left atrial appendage from the circulation was confirmed acutely and chronically by echocardiographic and angiographic evaluations. There was no device migration or damage to adjacent structures.

Conclusion: This novel device enables rapid, reliable, and safe exclusion of the left atrial appendage. The device provides a new therapeutic option for reducing the risk of stroke in patients with atrial fibrillation.

A trial fibrillation (AF) is associated with a 5-fold increase in the risk of stroke. ${ }^{1}$ located in the LAA, almost always in the distal, trabeculated portion. ${ }^{2,3}$ Exclusion of the LAA is a potential therapeutic option to reduce the risk of stroke in patients with AF, particularly in patients who cannot be anticoagulated with warfarin sodium., ${ }^{4,5}$

Accordingly, there is a need for a simple, minimally invasive technique that provides complete exclusion of the LAA. Recently, several novel techniques for LAA exclusion using thoracoscopic or percutaneous approaches have been reported. ${ }^{6-10}$ Although an epicardial approach to LAA exclusion has the potential advantages of simplicity (no transseptal puncture) and low procedural risk of embolism (no left heart endocardial catheters), there is currently no instrumentation available to facilitate rapid, reliable, and safe epicardial exclusion of the LAA.

A novel device, the atrial exclusion device (AED) (AtriCure, Inc, Cincinnati, Ohio), has been specifically designed for the exclusion of structures such as the LAA. This study was conducted to evaluate the ability of the AED to exclude the LAA during short and intermediate follow-up periods in a canine model.

\section{Material and Methods}

\section{AED Design}

The AED is composed of a rigid and a flexible member fabricated from $316 \mathrm{~L}$ stainless steel. The members are completely covered with braided polyester knit sheath to promote ingrowth. 


\section{Abbreviations and Acronyms \\ $\mathrm{AED}=$ atrial exclusion device \\ $\mathrm{AF}=$ atrial fibrillation \\ LA $=$ left atrial \\ LAA $=$ left atrial appendage \\ 2D EE = two-dimensional epicardial echocardiography}

The AED has an internal major diameter of $35 \mathrm{~mm}$ and a neutral spacing of approximately $1 \mathrm{~mm}$ between the steel members in the closed position (Figure 1). The AED has 2 configurations: open, in which distance between the members is greatest, and closed, in which the AED changes to a closed, flattened shape. In the open position, the AED is placed over the LAA and positioned at its base. The device is then closed, and the position is confirmed. Once the AED is in the closed position, the AED can be reopened and reapplied if necessary.

The polyester covering on the AED serves 2 functions. It promotes tissue ingrowth to securely anchor the AED onto the LAA without damage to left atrial (LA) tissue adjacent to the occlusion site, and it provides a soft covering for the AED to prevent trauma or erosion into surrounding anatomic structures such as the pulmonary artery and left ventricle.

\section{In Vivo Study}

Eight mongrel dogs (mean weight $29.1 \pm 4.0 \mathrm{~kg}$ ) were used in this study. This study was approved by the Cleveland Clinic's Institutional Animal Care and Use Committee, and all animals received humane care in compliance with the "Guide for the Care and Use of Laboratory Animals" prepared by the Institute of Laboratory Animal Resources, National Research Council, and published by the National Academy Press, revised 1996.

\section{Implant Study}

All surgical procedures were performed using sterile techniques. The animals were anesthetized with intravenous thiopental $(20 \mathrm{mg} / \mathrm{kg})$. Anesthesia was maintained with isoflurane (0.5\%-2.5\%). The animals were placed in the right lateral position. Electrocardiogram leads were attached to the extremities. The left carotid artery was used for continuous monitoring of arterial pressure.

A left fourth intercostal thoracotomy was performed, and the pericardium was opened to expose the LAA. A 14-gauge angiocatheter was inserted into the left upper pulmonary vein to monitor LA pressure and to inject contrast material for LA angiography in the 60-degree left anterior oblique and 30-degree right anterior oblique planes. Two-dimensional epicardial echocardiography (2D EE) was performed to confirm blood flow into the LAA before AED application. Hemodynamic data, including systemic arterial and LA pressures and heart rate, were also collected at baseline. Ventilatory support was transiently stopped during data-acquisition periods. Hemodynamic parameters were digitized in real time at a sampling rate of $200 \mathrm{~Hz}$ with a data-acquisition system (PowerLab, AD Instruments, Inc, Mountain View, Calif) and stored on a hard disk for subsequent analyses by a custom-made visual basic program on Excel software (Excel 2000, Microsoft Corporation, Redmond, Wash).
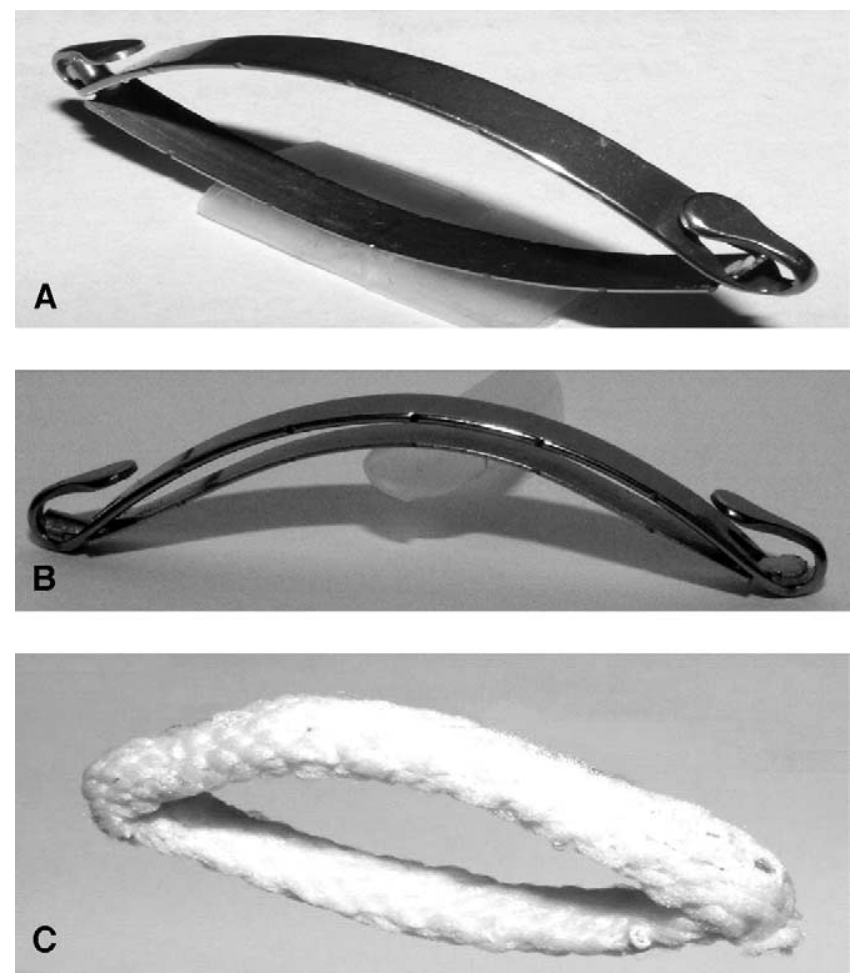

Figure 1. The atrial exclusion device (AED) consists of 2 stainless steel, flexible and rigid members, each of which is bar-shaped. Both members are covered with a braided polyester knit fabric. A, AED without polyester fabric in the open position. B, AED without polyester fabric in the closed position. C, AED with polyester fabric in the open position.

After all baseline data were obtained, the AED in the open position was placed over the end of the LAA and aligned with the base of the LAA. Once in the desired position, the AED was closed by gently squeezing the flexible and rigid members together. If AED deployment and position were not satisfactory, the AED was simply repositioned and reapplied in the proper location. After implantation, echocardiographic, angiographic, and hemodynamic data were obtained as before.

The chest was closed with a chest drain tube in place. Animals were followed for 7 days ( 2 dogs), 30 days ( $2 \operatorname{dogs}$ ), or 90 days (4 dogs).

\section{Explant Study}

On the day of the explant study, animals were placed under general anesthesia as for the implant study. The right femoral artery was used for continuous monitoring of arterial pressure. The chest was reentered through the original incision to expose the AED. Hemodynamic assessment, LA angiography, and 2D EE were performed in the same manner as during the implant study.

In one 7-day and two 90-day dogs, electrical stimulation was applied to the LAA remnant. Standard epicardial pacing wires were placed on the LA body and LAA remnant. LA pacing was performed in asynchronous mode to confirm that the heart could be 


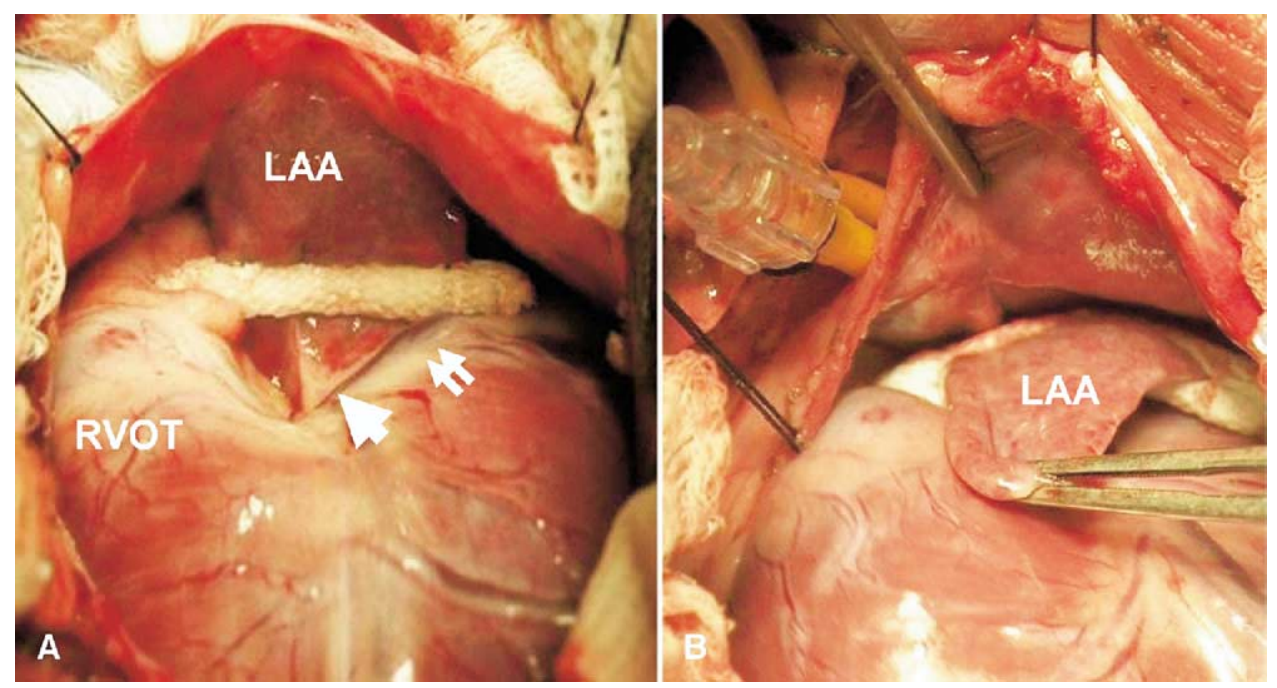

Figure 2. AED implanted at the base of the LAA (A, B). Surrounding structures such as main pulmonary artery, left circumflex artery (small arrows), and great cardiac vein (large arrow) were not affected by the AED (A). LAA, Left atrial appendage; RVOT, right ventricular outflow tract.

entrained. Then, stimulation $(20 \mathrm{~mA})$ was applied to 2 different locations on the LAA to attempt to pace the heart at a rate greater than the native sinus rate.

Before sacrifice, $500 \mathrm{U} / \mathrm{kg}$ of heparin was administered. Animals were then put to death by rapid intravenous injection of sodium pentobarbital $(50 \mathrm{mg} / \mathrm{kg})$ and potassium chloride $(120 \mathrm{mEq})$. The heart was excised with the AED in place, and the external and internal surfaces of the LA were photographed and examined. The heart was thoroughly investigated to evaluate the completeness of the LAA exclusion, and surrounding tissue and structures were examined for signs of damage and to evaluate tissue response.

\section{Histologic Evaluation}

Cardiac tissue specimens were excised from both sides of the AED for evaluation of tissue ingrowth into the polyester fabric coating. Tissue from the LAA distal to the implanted AED was also evaluated. The tissues were fixed in formalin for 48 hours and
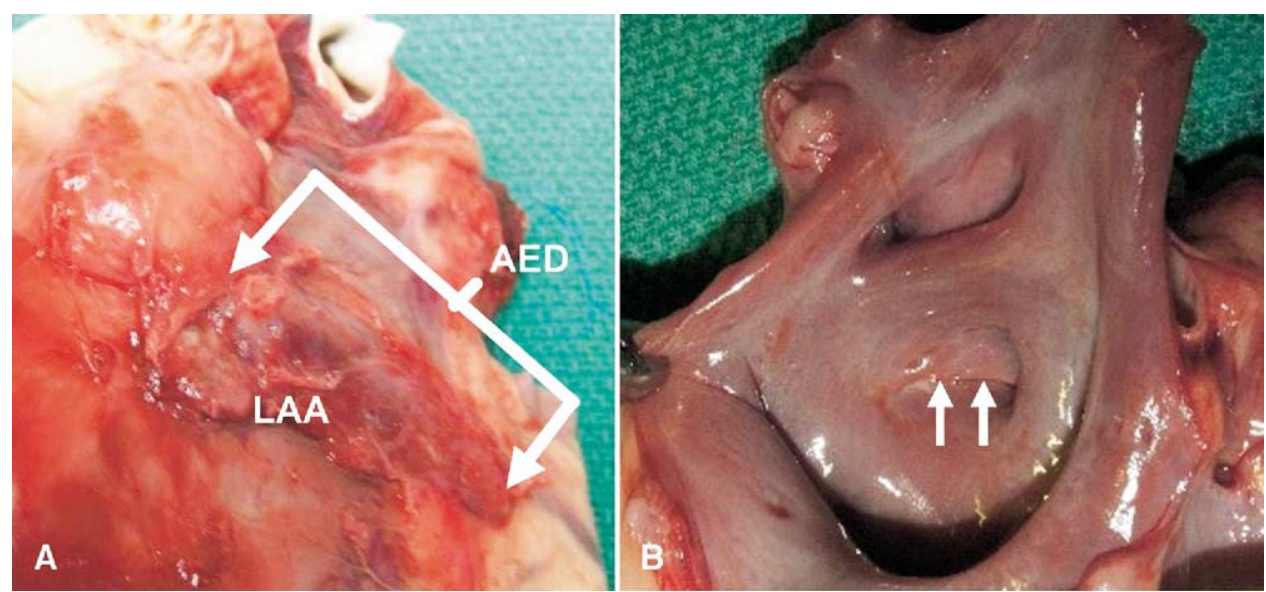

Figure 3. Gross anatomic views of the external LAA and the internal surface of the LAA orifice at 90 days. The entire AED was covered with clear pink tissue with no migration (A). Linear occlusion site (arrows) by the AED (B). $L A A$, Left atrial appendage; $A E D$, atrial exclusion device. 
prepared appropriately for evaluation. The sections were stained with hematoxylin-eosin.

\section{Data Analysis}

All values were expressed as mean \pm standard deviation. Repeatedmeasures analysis of variance was used to assess differences between data at preimplantation, postimplantation, and follow-up.

\section{Results}

\section{AED Implantation}

The AED was implanted on a beating heart in all animals without difficulty or hemodynamic change. AED application required approximately 15 seconds once the LAA was exposed. In 1 animal, initial AED position was unsatisfactory because the device was not at the base of the LAA; the AED was easily repositioned to ensure complete exclusion of the LAA (Figure 2). Postimplant 2D EE and LA angiography revealed no communication between the LAA and LA body in all animals. In addition, after implantation, Doppler echocardiography confirmed absence of blood flow into the LAA. All 8 dogs survived the procedure without devicerelated complications. Postoperative recovery was uneventful in all animals.

\section{AED Explantation}

At explantation, all AED clips remained positioned at the base of the LAA with no evidence of migration. There was no damage to adjacent cardiac structures; the LA body, circumflex coronary artery, and pulmonary artery were intact without erosion. In 7-day animals, there were mild adhesions between the AED and pericardium, and the LAA appeared intact. In all but one 90-day dog there were dense adhesions surrounding the AED, and the LAA had atrophied. In one 90-day animal, adhesions were only mild and the LAA was flaccid but not atrophied. Before explantation, 2D EE and LA angiography demonstrated no communication between the LAA and LA in all animals. Doppler echocardiography revealed no blood flow into the LAA in 7-day animals or in the one 90-day animal without atrophy of the LAA. Hemodynamic data revealed no significant changes among data at preimplantation (heart rate: $123 \pm 14.3$ beats $/ \mathrm{min}$, arterial pressure: $102 \pm 20.5 \mathrm{~mm} \mathrm{Hg}$, and LA pressure: $10.6 \pm 1.7$ $\mathrm{mm} \mathrm{Hg}$ ), postimplantation (heart rate: $124 \pm 16.4$ beats/ min, arterial pressure: $101 \pm 19.2 \mathrm{~mm} \mathrm{Hg}$, and LA pressure: $10.9 \pm 2.0 \mathrm{~mm} \mathrm{Hg}$ ), and follow-up (heart rate: $122 \pm 9.9$ beats/min, arterial pressure: $105 \pm 9.4 \mathrm{~mm} \mathrm{Hg}$, and LA pressure: $10.6 \pm 1.9 \mathrm{~mm} \mathrm{Hg}$ ). Pacing studies performed in one 7-day dog and two 90-day dogs confirmed electrical isolation of the LAA or LAA remnant.

\section{Gross and Histologic Examinations}

The heart was excised with the AED in place in all animals. Examination of the inside of the LA revealed a smooth endocardial surface at the origin of the LAA; there was no cul-de-sac, and there was no thrombus associated with the AED. In one 7-day animal, a 1-cm, firmly adherent LA thrombus was identified arising from the body of the LA directly opposite to the LAA orifice; this likely represented a site of endocardial damage caused by the catheter tip. There was no communication between the orifice of the LAA and the thrombus, indicating that there was no relationship between the thrombus formation and the AED application.

At 7 days, the distal LAA was intact. The polyester fabric was covered with a thin, pink tissue. Figure 3 shows a representative gross anatomic view of the external LAA and the internal surface of the LAA orifice at 90 days. Tissue ingrowth completely covered the AED and the LAA was atrophied in 3 of 4 dogs (Figure 3, A). In addition, the occlusion line was smooth and linear without any trabeculated portions (Figure 3, B). Hematoxylineosin-stained sections of the distal LAA tissue from non-atrophied LAAs showed myocardium with fibrotic remodeling and fatty infiltration, whereas the tissue from atrophied LAAs was completely replaced by fibrous tissue. In all cases, the polyester fabric was covered by fibrous connective tissue.

\section{Discussion}

\section{Key Findings}

A novel device applied to the epicardial surface completely excluded the LAA from the systemic circulation and did not migrate or cause damage to adjacent cardiac structures in the first 90 days after implantation. In addition, the AED provided electrical isolation of the LAA in 3 of 3 cases.

\section{AF, Stroke, and the LAA}

$\mathrm{AF}$ is responsible for $10 \%$ to $20 \%$ of strokes. Recent randomized trials have demonstrated that anticoagulation substantially reduces, but does not eliminate, the risk of stroke in patients with AF. ${ }^{1,11,12}$ However, anticoagulant therapy with warfarin is associated with both major (1\%-2\%/year) and minor (5\%-10\%/year) hemorrhagic complications. ${ }^{13,14}$ Because of the increased risk of anticoagulant-related complications with advancing age, many physicians are reluctant to prescribe anticoagulant therapy for elderly patients with AF. ${ }^{13,14}$ In addition, many patients are not compliant with anticoagulation. Because of these issues, recommended guidelines for anticoagulation are followed in less than half of outpatients with $\mathrm{AF}^{4,5}$

A potential alternative or adjunctive approach to stroke prevention in patients with AF is surgical management of the LAA. The LAA is the primary site of thrombus formation in patients with AF. Approximately $60 \%$ of LA thrombi in rheumatic AF form in the LAA, but this figure is $91 \%$ in patients with nonrheumatic $\mathrm{AF}^{2}{ }^{2}$ These reports led to the 
recommendation that the LAA be excised or excluded at the time of mitral valve surgery. ${ }^{15}$ This strategy has been associated with a reduction in the risk of late stroke in patients undergoing mitral valve surgery. ${ }^{16}$ In addition, early data suggest that LAA excision or exclusion reduces the risk of stroke in selected patients with nonvalvular $\mathrm{AF}^{2,17}$

\section{Techniques for LAA Management}

Cardiac surgeons have used a variety of techniques to manage the LAA. Suture ligation from the endocardial surface of the LA requires the use of cardiopulmonary bypass and may be associated with bleeding or injury to the circumflex coronary artery. In addition, endocardial suture ligation is incomplete in $10 \%$ to $30 \%$ of patients, and incomplete exclusion may predispose the patient to thromboembolic events. ${ }^{18-20}$ In contrast, external ligation or excision can be performed without opening the LA and without cardiopulmonary bypass. Such approaches may include suture ligation or use of cutting or noncutting surgical staplers. ${ }^{10,21}$ These techniques can cause troublesome bleeding if friable LA tissue tears. ${ }^{22}$ Epicardial application of a purse-string suture or loop may fail to occlude the LAA or may cause bunching of LA tissue; these might predispose to thrombus formation on the endocardial surface. In addition, these epicardial approaches, including this AED application, incur the potential risk of perioperative thromboembolism in patients with LAA thrombi that extend to the orifice of the LAA, because of the required manipulation of the LAA. Therefore, these approaches should be avoided in this patient population.

More recently, percutaneous approaches to LAA exclusion have been introduced. Several reports of preclinical data and human feasibility trials document successful endocardial application and the possibility of stroke prevention. ${ }^{6-9}$ However, residual flow around the occlusion device immediately after implant and at follow-up was observed in every patient, and the importance of this finding is uncertain. ${ }^{8}$

\section{The AED}

Unlike most surgical staplers, the AED has been developed specifically for exclusion of structures such as the LAA. It is configured for open surgical or thoracoscopic placement. It is easily placed on the beating heart and may be atraumatically repositioned if initial placement is unsatisfactory. When compared with epicardial stapling devices, the AED offers 2 advantages: (1) easy reapplication that ensures proper placement onto the base of the LAA and (2) elimination of the risk of bleeding that may originate along the occlusion (staple) line. The promotion of distal LAA atrophy by the AED, as indicated in our 30- and 90-day experiments, may eliminate the possibility of late recanalization of the LAA lumen, which is one of the potential problems of noncutting stapling devices. Angiography and echocardiography con- firmed complete exclusion of the LAA at 7, 30, and 90 days without damage to adjacent cardiac structures. The physical characteristics of the device and tissue ingrowth around the polyester covering prevented device migration. By 90 days, the LAA generally atrophied, leaving a smooth endocardial surface where its origin had been. Although there was no thrombus in the vicinity of the LAA, 1 animal did have a LA thrombus that was likely attributable to catheter-induced trauma at angiography. At present, the AED is further evolving to obtain confidence for clinical trial.

\section{Limitations}

These data include early follow-up in a relatively small number of healthy animals. None of the animals had AF, so the possibility of LA thrombus formation after AED application in the setting of AF cannot be excluded. However, the smooth endocardial surface at the origin of the LAA and the absence of a cul-de-sac in this region are very encouraging. This study does not test the hypothesis that exclusion of the LAA reduces the risk of stroke or other thromboembolism in patients or animals with AF.

\section{Further Clinical Implications}

This study suggested that the AED can occlude and isolate the LAA completely, safely, and rapidly without cardiopulmonary bypass and provide favorable biocompatibility over a mid-term period (90 days). The results may stimulate the development of novel device that allows for the AED deployment under a thoracoscopic approach.

To date, no report shows clear evidence of the role of LAA exclusion in the prevention of strokes for patients with nonvalvular AF. A randomized trial of LAA occlusion during routine coronary artery bypass surgery (Left Atrial Appendage Occlusion Study) is ongoing and will provide definitive evidence about the LAA exclusion in patients without mitral valve disease. ${ }^{23}$ Meanwhile, several reports have suggested that the LAA may be one of the sources of thrombus in patients with significant left ventricular dysfunction (eg, dilated cardiomyopathy), even in those who maintain sinus rhythm. ${ }^{24,25}$ If LAA exclusion is proven to reduce stroke risk in patients with nonvalvular AF and to be free from other complications, this novel device, the AED, may be suitable not only for patients with nonvalvular AF but also for the aforementioned high-risk patients in sinus rhythm for prevention of embolic events resulting from LAA thrombi.

\section{Conclusion}

The AED may provide an alternative or adjunctive therapeutic option for reducing the risk of stroke in patients with AF. 


\section{References}

1. Wolf PA, Abbott RD, Kannel WB. Atrial fibrillation as an independent risk factor for stroke: the Framingham Study. Stroke. 1991;22:983-8.

2. Blackshear JL, Odell JA. Appendage obliteration to reduce stroke in cardiac surgical patients with atrial fibrillation. Ann Thorac Surg. 1996; 61:755-9.

3. Al-Saady NM, Obel OA, Camm AJ. Left atrial appendage: structure, function, and role in thromboembolism. Heart. 1999;82:547-54.

4. Stafford RS, Singer DE. National pattern of warfarin use in atrial fibrillation. Arch Intern Med. 1996;156:2537-41.

5. Stafford RS, Singer DE. Recent national pattern of warfarin use in atrial fibrillation. Circulation. 1998;97:1231-3.

6. Nakai T, Lesh MD, Gerstenfeld EP, Virmani R, Jones R, Lee RJ. Percutaneous left atrial appendage occlusion (PLAATO) for preventing cardioembolism: first experience in canine model. Circulation. 2002;105:2217-22.

7. Sievert H, Lesh MD, Trepels T, Omran H, Bartorelli A, Bella PD, et al. Percutaneous left atrial appendage transcatheter occlusion to prevent stroke in high-risk patients with atrial fibrillation: early clinical experience. Circulation. 2002;105:1887-9.

8. Hanna IR, Kolm P, Martin R, Reisman M, Gray W, Block PC. Left atrial structure and function after percutaneous left atrial appendage transcatheter occlusion (PLAATO): six-month echocardiographic follow-up. J Am Coll Cardiol. 2004;43:1868-72.

9. Meier B, Palacios I, Windecker S, Rotter M, Cao QL, Keane D, et al. Transcatheter left atrial appendage occlusion with Amplatzer devices to obviate anticoagulation in patients with atrial fibrillation. Catheter Cardiovasc Interv. 2003;60:417-22.

10. Odell JA, Blackshear JL, Davies E, Byrne WJ, Kollmorgen CF, Edward WD, et al. Thoracoscopic obliteration of the left atrial appendage: potential for stroke reduction? Ann Thorac Surg. 1996;61: 565-9.

11. Gage BF, Waterman AD, Shannon W, Boechler M, Rich MW, Radford MJ. Validation of clinical classification schemes for predicting stroke: results from the National Registry of Atrial Fibrillation. JAMA. 2001;285:2864-70.

12. Kannel WB, Wolf PA, Benjamin EJ, Levy D. Prevalence, incidence, prognosis, and predisposing conditions for atrial fibrillation: populationbased estimates. Am J Cardiol. 1998;82:2N-9N.

13. Levine MN, Raskob G, Landefeld S. Hemorrhagic complications of anticoagulant treatment. Chest. 2001;119:108S-21S.

14. Levine MN, Raskob G, Beyth RJ, Kearon C, Schulman S. Hemorrhagic complications of anticoagulant treatment. the seventh ACCP conference on antithrombotic and thrombolytic therapy. Chest. 2004; 126:287S-310S.

15. Bonow RO, Carabello B, de Leon Jr AC, Edmunds Jr LH, Fedderly BJ, Freed MD, et al. ACC/AHA guidelines for the management of patients with valvular heart disease: A report of the American College of Cardiology/American Heart Association Task Force on practice guidelines (Committee on Management of Patients with Valvular Heart Disease). J Am Coll Cardiol. 1998;32:1486-582.

16. García-Fernández MA, Pérez-David E, Quiles J, Peralta J, GarcíaRojas I, Bermejo J, et al. Role of left atrial appendage obliteration in stroke reduction in patients with mitral valve prosthesis: a transesophageal echocardiographic study. J Am Coll Cardiol. 2003;42:1253-8.

17. Johnson WD, Ganjoo AK, Stone CD, Srivyas RC, Howard M. The left atrial appendage: our most lethal human attachment! Surgical implications. Eur J Cardiothorac Surg. 2001;17:718-22.

18. Katz ES, Tsiamtsiouris T, Applebaum RM, Schwartzbard A, Tunick PA, Kronzon I, et al. Surgical left atrial appendage ligation is frequently incomplete: a transesophageal echocardiographic study. J Am Coll Cardiol. 2000;36:468-71.

19. Fisher DC, Tunick PA, Kronzon I. Large gradient across a partially ligated left atrial appendage. J Am Soc Echocardiogr. 1998;11:1163-5.

20. Rosenzweig BP, Katz E, Kort S, Schloss M, Kronzon I. Thromboembolus from a ligated left atrial appendage. J Am Soc Echocardiogr. 2001;14:396-8.

21. DiSesa V, Tam S, Cohn LH. Ligation of left atrial appendage using an automatic surgical stapler. Ann Thorac Surg. 1988;46:652-3.

22. Gillinov AM, Pettersson G, Cosgrove DM. Stapled excision of the left atrial appendage. J Thorac Cardiovasc Surg. 2005;129:679-80.

23. Crystal E, Lamy A, Connolly SJ, Kleine P, Hohnloser SH, Semelhago L, et al. Left Atrial Appendage Occlusion Study (LAAOS): a randomized clinical trial of left atrial appendage occlusion during routine coronary artery bypass graft surgery for long-term stroke prevention. Am Heart J. 2003;145:174-8.

24. Vigna C, Russo A, De Rito V, Perna G, Villella A, Testa M, et al. Frequency of left atrial thrombi by transesophageal echocardiography in idiopathic and in ischemic dilated cardiomyopathy. Am J Cardiol. 1992;70:1500-1.

25. Siostrzonek P, Koppensteiner R, Gössinger H, Zangeneh M, Heinz G, Kreiner $\mathrm{G}$, et al. Hemodynamic and hemorheologic determinants of left atrial spontaneous echo contrast and thrombus formation in patients with idiopathic dilated cardiomyopathy. Am Heart J. 1993;125: 430-4. 